\title{
O Prêmio Nobel de Física de $2013^{+*}$
}

José Maria Filardo Bassalo

Academia Paraense de Ciências

Belém - PA

\section{Resumo}

Neste artigo, trataremos do Prêmio Nobel de Física de 2013, concedido aos físicos, o inglês Peter Ware Higgs e o belga François Englert pela previsão do bóson de Higgs, partícula responsável pela massa das partículas elementares.

Palavras-chave: Prêmio Nobel de Física de 2013. Higgs e Englert. Bóson de Higgs.

\begin{abstract}
In this article, we will address the 2013 Nobel Prize in Physics awarded to the physicists, the englishman Peter Ware Higgs and the belgian François Englert for the prediction of the Higgs boson, the particle responsible for the mass of the elementary particles.
\end{abstract}

Keywords: 2013' Physics Nobel Prize. Higgs and Englert. Higgs Boson.

\section{Peter Ware Higgs}

O Prêmio Nobel de Física (PNF) de 2013 foi concedido aos físicos, o inglês Peter Ware Higgs (n.1929) e o belga François Englert (n.1932) pela "desco-

\footnotetext{
${ }^{+}$The 2013 Physics Nobel Prize

* Recebido: dezembro de 2013. Aceito: fevereiro de 2013.
} 
berta teórica de um mecanismo que contribui para o entendimento da origem da massa das partículas subatômicas". A partícula decorrente desse mecanismo, conhecida então como bóson de Higgs, prevista em 1964, teve uma evidência de $3 \sigma$ (o que não garante sua confirmação, como veremos mais adiante) em experiências realizadas por colaborações dos dois principais aceleradores do mundo: o Tevatron New Phenomena \& Higgs Working Group: Combined D0/CDF do Fermi National Accelerator Laboratory (FERMILAB), nos Estados Unidos, e A Toroidal LHC ApparatuS (ATLAS) e Compact Muon Solenoid (CMS) do Large Hadron Collider (LHC), do Conseil Européen pour la Recherche Nucléaire (hoje: European Organization for Nuclear Research) (CERN), na Europa, e, finalmente, ela foi anunciada em julho de 2012, com um nível de confiança de $5 \sigma$, que é um critério internacional sobre a existência de uma partícula, como veremos no decorrer deste artigo.

Higgs nasceu em 29 de maio de 1929 em Elswick, distrito de Newcastle upon Tyne, na Inglaterra. Devido à asma, que adquiriu na infância, seus primeiros estudos foram realizados praticamente em casa. Depois que seu pai, engenheiro de som da British Broadcasting Corporation ("Corporação Britânica de Radiodifusão") (BBC), se transferiu para Bedford, perto de Bristol, Higgs estudou, entre 1941 e 1946, na Cotham Grammar School, em Bristol, que se tornara célebre por ter o físico inglês Paul Adrien Maurice Dirac (1902-1984; PNF, 1933) (BASSALO; CARUSO, Dirac, Livraria da Física, 2013) como um de seus alunos.

Com 17 anos de idade, em 1946, Higgs foi estudar na City of London School, especializando-se em Matemática. Logo depois, em 1947, ele entrou para o King College, em Londres, graduando-se em Física, em 1950, como o primeiro aluno de sua classe; em 1952, obteve o Mestrado em Física, e começou a realizar seu doutoramento no grupo de Física Molecular, trabalhando com os químicos teóricos ingleses Charles Alfred Coulson (1910-1974) (também matemático aplicado) e Hugh Christopher Longuet-Higgins (1923-2004), sendo este aluno de doutorado de Coulson na Universidade de Oxford. Higgs defendeu sua Tese de Doutorado, em 1954, intitulada Some Problems in the Theory of Molecular Vibrations ("Alguns Problemas na Teoria das Vibrações Moleculares"), sob a orientação de Coulson. Entre 1954 e 1956, Higgs foi Pesquisador Sênior da University of Edinburgh (UE), voltando a essa Universidade, em 1960, para ser Lecturer no Tait Institute of Mathematical Physics, depois de trabalhar no Imperial College London e University College. Em 1980, assumiu a Cadeira de Física da UE e, em 1983, tornou-se Membro (Fellow) da Royal Society of Edinburgh (RSE) e da Royal Society of London (RSL) e, em 1991, do Institute of Physics (IP). Aposentou-se da UE, em 1996, ocasião em que recebeu o título de Professor Emérito. 
Destaque-se que, antes de compartilhar o PNF2013, Higgs recebeu outras honrarias, tais como: em 1981, Hughes Medal da RSL; em 1984, Rutherford Medal and Prize do Institute of Physics (IP); em 1997, Dirac Medal and Prize do IP e High Energy and Particle Physics Prize da European Physical Society (EPS) [este, com os físicos belgas Englert e Robert Brout (1928-2011)]; em 2004, Wolf Prize in Physics (com Englert e Brout) pela Wolf Foundation; em 2008, foi retratado pelo artista inglês Ken Currie (n.1960); em 2009, Oskar Klein Memorial Lecture Medal da Royal Swedish Academy of Sciences (RSAS); em 2010, J. J. Sakurai Prize for Theoretical Particle Physics [com Englert, Brout e os físicos, os norte-americanos Gerald Stanford Guralnik (n.1936) e Carl Richard Hagen (n.1937), e o indianoinglês Thomas Walter Bannerman Kibble (n.1932)] da American Physical Society (APS); em 2011, Edinburgh Award da City of Edinburgh Council; e, em 2012, Higgs Medal da RSE. Por fim, em 06 de julho de 2012, a UE criou o The Higgs Center for Theoretical Physics e a Chair Peter Higgs. Aliás, é interessante registrar que, como Higgs não tem telefone celular e quase não usa o seu fixo, ele soube que havia compartilhado o PNF2013, por intermédio de uma mulher que o abordou em uma rua em Edinburgh e o congratulou (wikipedia/Higgs). Note-se que, no dia 08 de dezembro de 2013, Higgs apresentou sua Nobel Lecture: Evading the Goldstone Theorem ("Contornando o Teorema de Goldstone").

\section{François Englert}

O Barão Englert nasceu no dia 06 de novembro de 1932, em Etterbeck, Bruxelas, na Bélgica. Com a ocupação da Bélgica pelo Exército Nazista, por ocasião da Segunda Guerra Mundial (SGM) (1939-1945), ele teve de ocultar sua descendência judaica e viver em orfanatos e casas para crianças nas cidades de Dinant, Lustin, Stoumont e Annevoie-Rouillion, cidades essas que foram, em 1945, libertadas pelo Exército Norte-Americano.

De volta a Bruxelas, ele se graduou em Engenharia Eletromecânica, em 1955, na Universidade Livre de Bruxelas (ULB) onde, também, obteve o Doutorado em Física, em 1959. De 1959 até 1961, Englert foi para a Cornell University, nos Estados Unidos, trabalhando com Brout. Em 1961, Englert voltou à ULB e tornou-se professor universitário. Em 1980, juntamente com Brout, criou um grupo para trabalhar em Física Teórica. Em 1984, foi indicado para a "Cadeira" ou "Cátedra" Professor Sackler Chair, na School of Physics and Astronomy da Tel-Aviv University. Em 1998, Englert foi indicado como Professor Emérito da ULB. Por fim, em 2011, o Institute for Quantum Studies da Chapman University (Orange, Califórnia), ofereceu-lhe o cargo de Eminente Professor Visitante. 
Destaque-se que, antes de compartilhar o PNF2013, Englert recebeu outras honrarias, tais como: em 1978, First Prize (com Brout e o físico belga Edgard Gunzig) da Gravity Research Foundation Awards; em 1982, Francqui Prize da Francqui Foundation; em 1997, High Energy and Particle Physics Prize (com Brout e Higgs) da European Physical Society (EPS); em 2004, Wolf Prize in Physics (com Brout e Higgs), pela Wolf Foundation; em 2010, J. J. Sakurai Prize for Theoretical Particle Physics (com Brout, Guralnik, Hagen, Higgs e Kibble). Por fim, em 08 de julho de 2013, o Rei Alberto II da Bélgica (n.1934), por Decreto Real, concedeu a Englert o Título Nobre de Barão. (wikipedia/Englert). Registre-se que, no dia 08 de dezembro de 2013, Englert apresentou sua Nobel Lecture: The BEH Mechanism and Its Scalar Boson ["O Mecanismo BEH (Brout-EnglertHiggs) e Seu Bóson Escalar].

\section{Resumo histórico sobre as Partículas Elementares}

Agora, vejamos o significado dessa premiação e, para isso, vamos fazer um resumo histórico sobre as Partículas Elementares. Para os antigos filósofos gregos, existiam quatro tipos de forças: as que atuam nos corpos nas proximidades de nosso planeta Terra (as terrestres); as que atuam nos corpos celestes (as celestes); as exercidas pela magnetita ou ímã natural (hoje conhecida quimicamente como o óxido de ferro: $\mathrm{Fe}_{3} \mathrm{O}_{4}$ ) - a força magnética; e as exercidas pelo âmbar, quando este é atritado com um pedaço de lã - a força elétrica. Estas duas últimas foram mencionadas pelo filósofo e astrônomo grego Tales de Mileto (624-546). Durante longo tempo, os fenômenos elétricos e magnéticos, por se apresentarem muito semelhantes, foram confundidos, até serem, pela primeira vez, diferenciados pelo matemático italiano Ge(i)rolano Cardano (Jerome Cardan) (1501-1576). Observação análoga foi realizada pelo médico e físico inglês William Gilbert (15441603), que a registrou em seu famoso tratado De Magnete, publicado em 1600. Aliás, nesse livro, esse físico cunhou o termo elétrico para os corpos que se comportavam como o âmbar (elektron, em grego) quando atritado com lã. Tais forças, elétrica e magnética, só foram unificadas no Século 19, conforme veremos mais adiante.

As duas primeiras forças relacionadas acima, terrestres e celestes, discutidas pelo filósofo grego Aristóteles de Siracusa (384-322) em seus Livros V-VIII, Física (Les Belles Lettres, 1996), teve sua primeira ideia de unificação considerada pelo astrônomo armeno Abu Ar-Rayan Muhammad ibn Ahmad al-Biruni (973c.1051) ao afirmar que os fenômenos físicos no Sol, na Terra e na Lua obedecem às mesmas leis [Abdus Salam, IN: Em Busca da Unificação (Gradiva, 1991)]. 
Mais tarde, em 1602, o físico, matemático e astrônomo italiano Galileu Galilei (1564-1642) afirmou que as leis que regem as forças terrestres e celestes são universais [José Leite Lopes, Albert Einstein e a Imagem Física do Mundo, CBPFCS-011/97 (Abril de 1997)]. Contudo, foi o físico inglês Sir Isaac Newton (16421727) quem formalizou essa unificação por intermédio de sua célebre Lei da Gravitação Universal, apresentada no terceiro livro de seu famoso tratado intitulado Philosophiae Naturalis Principia Mathematica ("Princípios Matemáticos da Filosofia Natural"), publicado em $1687^{1}$.

Por sua vez, as primeiras experiências indicando a unificação entre as forças elétrica e magnética foram realizadas pelo farmacêutico e físico dinamarquês Hans Christiaan Oersted (1777-1851). Com efeito, em 1807, Oersted procurou, sem êxito, encontrar uma relação entre aquelas forças. Ela só foi por ele encontrada no inverno de 1819-1820, quando ministrou, na Universidade de Copenhague, um curso sobre Eletricidade, Galvanismo e Magnetismo. Durante esse curso, Oersted realizou uma série de experiências. Por exemplo, em fevereiro de 1820, observou que um condutor esquentava quando era percorrido por uma corrente elétrica. Também, nessas experiências, Oersted procurou encontrar uma relação entre eletricidade e magnetismo, examinando o que acontecia com uma agulha magnética ao ser colocada perpendicularmente ao fio condutor do circuito galvânico utilizado. No entanto, não registrou nenhum movimento perceptível da agulha. Porém, ao término de uma aula noturna daquele curso, no começo de abril de 1820, ocorreulhe a ideia de colocar o fio condutor paralelamente à direção da agulha magnética; aí, então, percebeu uma razoável deflexão dessa agulha, e a procurada relação entre o magnetismo e o "galvanismo" estava, então, descoberta. Observe-se que essa descoberta foi relatada ao físico e químico inglês Michael Faraday (1791-1867), em carta escrita pelo físico e astrônomo holandês Christopher Hansteen (17841873), então assistente de Oersted. É oportuno registrar que, no início do Século 19, era hábito distinguir o estudo da "eletricidade estática" do estudo das correntes elétricas ("galvanismo"), cujas primeiras pesquisas foram conduzidas pelo fisiologista italiano Luigi Galvani (1737-1798), em 1786, e pelo físico italiano Alessandro Giuseppe Volta (1745-1827), em 1794, ocasião em que este cientista cunhou o termo "galvanismo". É ainda interessante observar que a criação de um campo magnético por uma corrente elétrica foi também confirmada, ainda em 1820, em

${ }^{1}$ Great Books of the Western World 12. Encyclopaedia Britannica, Inc., 1993. 
experiências realizadas pelos físicos franceses Dominique François Jean Arago (1786-1853) ${ }^{2}$ e André Marie Ampère (1775-1836) ${ }^{3}$.

Uma vez encontrada a relação entre as forças elétrica e magnética, uma nova relação precisava ser pesquisada, qual seja, entre o "eletromagnetismo" (termo cunhado por Ampère) e a gravitação. Um dos primeiros a realizar experiências nesse sentido foi Faraday. Contudo, em 1849, ele escreveu em seu Diário de Laboratório que não havia conseguido mostrar que a gravidade poderia induzir correntes elétricas em peças de metal que caiam do topo de uma sala de aula na Royal Institution of Great Britain ${ }^{4}$. Outra tentativa de encontrar aquela mesma relação, e igualmente frustrada, foi apresentada pelo matemático alemão Georg Friedrich Bernhard Riemann (1826-1866), um pouco antes de morrer ${ }^{5}$.

A formulação matemática da unificação entre as forças elétrica e magnética - conhecida desde então como força eletromagnética - foi finalmente conseguida pelo físico e matemático escocês James Clerk Maxwell (1831-1879), em seu livro intitulado A Treatise on Electricity and Magnetism, publicado em 1873 (Dover, 1954). Aliás, é oportuno dizer que, nesse livro, Maxwell apresentou também a unificação da Óptica com o Eletromagnetismo ao demonstrar que a luz é uma onda eletromagnética.

Note-se que o Século 19 terminou com a ideia de que só existiam duas forças distintas na Natureza: a gravitacional newtoniana e a eletromagnética maxwelliana, em virtude das tentativas frustradas de unificá-las, conforme destacamos acima.

No Século 20, uma nova tentativa de unificar o eletromagnetismo com o campo escalar gravitacional foi apresentada pelo físico finlandês Gunnar Nordström (1881-1923), em 19146. Mais tarde, em 19187 , o matemático e físico

${ }^{2}$ Annales de Chimie et de Physique, v. 15, p. 93, 1820.

${ }^{3}$ Annales de Chimie et de Physique, v. 15, p. 170, 1820.

MARTINS, R. de A. Cadernos de História e Filosofia da Ciência, v. 10, p. 87, UNICAMP, 1986.

WHITTAKER, E. T. A History of the Theories of Aether and Electricity: The Classical Theories. Thomas Nelson and Sons Ltda., 1951.

${ }^{4}$ PAIS, A. 'Subtle is the Lord...' The Science and the Life of Albert Einstein. Oxford University Press, 1982.

${ }^{5}$ MISNER, C. W.; THORNE, K. S.; WHEELER, J. A. Gravitation. W. H. Freeman and Company, 1973.

${ }^{6}$ Zeitschrift für Physik, v. 15, p. 504, 1914. 
alemão Hermann Klaus Hugo Weyl (1885-1955) tentou essa unificação baseandose na generalização espaço-temporal da geometria riemanniana. Em 1919, inspirado nesse trabalho de Weyl, o matemático e linguista alemão Theodor Kaluza (1885-1954) discutiu com o físico germano-suíço-norte-americano Albert Einstein (1879-1955; PNF, 1921) uma nova possibilidade de unificar o eletromagnetismo com a gravitação, por intermédio de uma generalização da Teoria Geral da Relatividade (TGR) [esta havia sido desenvolvida por Einstein, em $1915^{8}$, segundo a qual a gravitação é decorrência da curvatura do espaço-tempo]. Para Kaluza, a TGR poderia ser generalizada para um espaço de cinco (5) dimensões, na qual a quinta dimensão era comprimida em um pequeno círculo. Desse modo, as equações de Einstein do campo gravitacional, escritas em cinco dimensões, reproduziriam as usuais equações einsteinianas em quatro dimensões, acrescidas de um conjunto de equações que representam as equações de Maxwell do campo eletromagnético. Provavelmente na conversa referida acima, Einstein tenha discutido com Kaluza sua ideia de que as partículas eletrizadas eram mantidas juntas por forças gravitacionais, segundo seus artigos publicados também em 1919 ${ }^{9}$. Aliás, nesses artigos, Einstein usou a ideia da constante cosmológica $\Gamma$ [proposta em $1917^{10}$, para manter estático o Universo] e procurou um vínculo entre a gravitação e o eletromagnetismo. Registre-se que, em 1921, Einstein apresentou o trabalho de Kaluza à Academia Prussiana de Ciências, sendo então publicado em seus Anais $^{11}$, ainda em 1921. Também em $1921^{12}$, o astrônomo inglês Sir Arthur Stanley Eddington (1882-1944) publicou um artigo no qual propôs a unificação entre a gravitação e o eletromagnetismo seguindo a mesma ideia de Weyl.

Em $1923^{13}$, com a colaboração do físico alemão Jakob Grommer (18791933), Einstein escreveu um trabalho no qual estudaram as soluções de singulari-

${ }^{7}$ Sitzungsberichte Preussische Akademie der Wissenschaften, Part 1, p. 465, 1918.

${ }^{8}$ Sitzungsberichte Preussische Akademie der Wissenschaften, v. 2, p. 778; 799; 831;844, 1915 .

${ }^{9}$ Sitzungsberichte Preussische Akademie der Wissenschaften, Part 1, p. 349; 463, 1919.

${ }^{10}$ Sitzungsberichte Preussische Akademie der Wissenschaften, v. 1, p. 142, 1917.

${ }^{11}$ Sitzungsberichte Preussische Akademie der Wissenschaften, Part 1, p. 966, 1921.

${ }^{12}$ Proceedings of the Royal Society of London, v. 99, p. 104, 1921.

${ }^{13}$ Scripta Jerusalem Universitat, v. 1, n. 7, 1923. 
dades-livres da Teoria de Kaluza. Ainda em $1923^{14}$, Einstein apresentou um esboço não-matemático de uma generalização da geometria riemaniana, na qual englobaria em um campo total, conhecido desde então como campo unificado, os campos gravitacional e eletromagnético.

Mais tarde, em 1926 ${ }^{15}$, o físico sueco Oskar Benjamin Klein (1894-1977) contornou a dificuldade apresentada pela Teoria de Kaluza, afirmando que a não observação da quinta dimensão kaluziana devia-se ao fato de que o raio do pequeno círculo considerado naquela teoria era da ordem de $10^{-33} \mathrm{~cm}$, o chamado comprimento de Planck $\left(\ell_{\mathrm{P}}=\sqrt{\hbar \mathrm{G} / \mathrm{c}^{3}}\right)$, comprimento esse correspondente à energia de $10^{19} \mathrm{GeV}$, conhecida como energia de Planck $\left(\mathrm{M}_{\mathrm{P}} \mathrm{c}^{2}=\sqrt{\hbar \mathrm{c}^{5} / G}\right)$, em que c é a velocidade da luz no vácuo, $\mathrm{M}_{\mathrm{P}}=10^{-5} \mathrm{~g}$ é a massa de Planck e G é a constante da gravitação universal (Newton-Cavendish).

A unificação entre as forças da Natureza até então conhecidas (gravitacional e eletromagnética) tornou-se mais complicada com a descoberta, na década de 1930, de mais duas forças, a fraca e a forte, decorrentes da radioatividade. Vejamos como aconteceu a descoberta da radioatividade. Na sessão do dia 20 de janeiro de 1896 da Academia Francesa de Ciências, o matemático e físico francês Henri Poincaré (1854-1912) apresentou as primeiras fotografias de raios- $\boldsymbol{X}$ enviadas pelo físico alemão Wilhelm Conrad Roentgen (1845-1923; PNF, 1901), cuja descoberta dos mesmos havia sido encontrada por ele, em $1895^{16}$.

Presente a essa sessão, o físico francês Antoine Henry Becquerel (18521908) perguntou a Poincaré de que parte do tubo de Hittorf (1869) [também conhecido como tubo de Geissler (1855) e tubo de Crookes (1874/1875), que produzem raios catódicos] que Roentgen utilizara, haviam saído esses raios. "Da parte oposto ao catodo, que se tornara fluorescente", respondeu Poincaré. Sendo especialista em luminescência (fluorescência e fosforescência), especialidade essa que aprendera com seu avô e seu pai, respectivamente, os físicos franceses Antoine César (1788-1878) e Edmond (1820-1891), Henri passou a realizar experiências procurando uma relação entre as substâncias fluorescentes e a emissão dos raios- $\boldsymbol{X}$

${ }^{14}$ Sitzungsberichte Preussische Akademie der Wissenschaften, p. 32; 76; 137; Nature, v. 112, p. 448, 1923.

${ }^{15}$ Zeitschrift für Physik, v. 37, p. 895, 1926; Nature, v. 118, p. 516, 1926.

${ }^{16}$ Sitzungsberichte der Würzburger Physikalischen-Medicinischen Gesellschaft, p. 132, 1895.

Bassalo, J. M. F. 
por parte das mesmas. Não encontrou tal relação, no entanto, descobriu um novo fenômeno físico: a radioatividade.

Em fevereiro de 1896, Henri observou que cristais de sulfato de urâniopotássio [uranilo $\left(\mathrm{UO}_{2}\right)$ ] eram capazes de impressionar uma chapa fotográfica recoberta com papel escuro, estando o conjunto exposto à luz solar. Como na primeira experiência que realizou, havia submetido o conjunto aos raios solares, a explicação que deu para o fato de haver sido impressionada a chapa fotográfica, foi a de que a luz solar havia provocado fluorescência nos cristais com a emissão de raios- $\boldsymbol{X}$ que, por sua vez, atravessaram o papel escuro que envolvia os cristais, indo, por conseguinte, impressionar a chapa fotográfica.

Em outra experiência, realizada no dia 01 de maio de 1896 e, desta vez, sem uso da luz solar (provavelmente em um dia chuvoso de Paris), o fenômeno se repetiu. Henri concluiu então que o composto emitia certos "raios" descobrindo, dessa forma, um novo fenômeno físico. Por essa descoberta, compartilhou com o Casal Curie [o químico e físico francês Pierre (1859-1906) e a química e física polonesa Marya (Marie) Sklodowska (Madame Curie) (1867-1934)], o Prêmio Nobel de Física (PNF) de 1903. Registre-se que essa descoberta de Becquerel foi por ele publicada em $1896^{17}$, e que o nome radioatividade foi dado por Madame Curie, em 1898, ao observar que o tório (Th) se comportava como o uranilo de Becquerel.

A descoberta dos raios- $\boldsymbol{X}$ por Roentgen, em 1895, conforme vimos acima, levou o físico inglês Ernest Rutherford, Lord Rutherford de Nelson (1871-1937) a medir a ionização provocada por esses raios, trabalho esse que fez como colaborador do físico inglês Sir Joseph John Thomson (1856-1940; PNF, 1906) - que viria a descobrir o elétron ( $\mathrm{e}^{-}$), em $1897^{18}$ - de quem Rutherford era pesquisadorestudante no Laboratório Cavendish, em Londres, e publicado em $1896^{19}$. Por outro lado, a descoberta da radioatividade por Henri Becquerel, em 1896, referida anteriormente, conduziu Rutherford a outro aspecto de suas pesquisas, qual seja, a de medir a ionização provocada pelos "raios de Becquerel" fazendo-os passar através de folhas metálicas. Neste seu trabalho, descobriu, em $1898^{20}$, que os "rai-

${ }^{17}$ Comptes Rendus Hebdomadaires des Séances de l'Academie des Sciences de Paris, (CRHSASP), v. 122, p. 420; 501, 1896.

18

Philosophical Magazine, v. 44, p. 295, 1897.

${ }^{19}$ Philosophical Magazine,v. 42, p. 392, 1896.

${ }^{20}$ Proceedings of the Cambridge Philosophical Society, v. 9, p. 401, 1898. 
os Becquerel" eram constituídos de dois tipos de partículas: alfa $(\alpha)$, carregada positivamente, e beta ( $\beta$ ), carregada negativamente. Em 1899, em trabalhos independentes, Becquerel21, os físicos austríacos Stefan Meyer (1872-1949) e Egon Ritter von Schweidler (1873-1948) ${ }^{22}$, e o físico alemão Frederick Otto Giesel $(1852-1927)^{23}$ observaram a deflexão magnética sofrida por essas partículas. Em $1900^{24}$, Becquerel mostrou que os raios $\beta$ eram raios catódicos, isto é, elétrons. Ainda em $1900^{25}$, o físico francês Paul Villard (1860-1934) observou que a radioatividade possuía uma terceira parcela que não era defletida pelo campo magnético, parcela essa penetrante e semelhante aos raios- $\boldsymbol{X}$, à qual Rutherford denominou de gama $(\gamma)$. Essa descoberta foi confirmada por Becquerel, também em 190026.

Depois do sucesso de seu trabalho sobre a radioatividade, Rutherford passou a usá-la, principalmente a radioatividade alfa $(\alpha)$, em outros experimentos, agora com a colaboração dos físicos, o alemão Hans (Joahnnes) Wilhelm Geiger (1882-1945) e o inglês Ernst Marsden (1889-1970), sobre o espalhamento de partículas $\alpha$ pela matéria. Com efeito, em $1906^{27}$, Rutherford apresentou os resultados de experiências nas quais observou um pequeno espalhamento (desvio de aproximadamente $2^{0}$ ) de partículas $\alpha$ ao passarem através de uma lâmina de mica de $0,003 \mathrm{~cm}$ de espessura. Em $1908^{28}$, Geiger estudou o espalhamento de um feixe de partículas $\alpha$, oriundo de um composto de rádio, o brometo de rádio $\left(\operatorname{RaBr}_{2}\right)$, através de uma lâmina fina de metal [alumínio $(\mathrm{A} \ell)$ e ouro $(\mathrm{Au})$ ]. As partículas $\alpha$ [que são núcleos de hélio $\left({ }_{2} \mathrm{He}^{4}\right)$, conforme Rutherford e o químico inglês Thomas Royds (1884-1955) mostraram em 190929] espalhadas eram detectadas em contadores de cintilações. Usando essa técnica de contagem, Geiger e Marsden, em

${ }^{21}$ CRHSASP 129, p. 996, 1899.

${ }^{22}$ Physikalische Zeitschrift, v. 1, p. 113, 1899.

23

Annalen der Physik, v. 69, p. 834, 1899.

${ }^{24}$ CRHSASP 130, p. 809, 1900.

${ }^{25}$ CRHSASP 130, p. 1010; p. 1178, 1900.

${ }^{26}$ CRHSASP 130, p. 1154, 1908.

${ }^{27}$ Philosophical Magazine, v. 11, p. 166; 12, p. 134, 1906.

${ }^{28}$ Proceedings of the Royal Society of London, A81, p. 174, 1908.

29

Philosophical Magazine, v. 17, p. 281, 1909. 
$1909^{30}$, estudaram o espalhamento de um feixe de partículas $\alpha$ [oriundas do radônio (Rn)], através de uma lâmina fina de metal. Nesse estudo, eles observaram que do feixe não muito bem colimado e contendo cerca de 8.000 daquelas partículas, apenas uma delas era refletida, ou seja, era espalhada num ângulo $>90^{\circ}$. Este tipo de espalhamento foi também comentado por Geiger, em 1910 ${ }^{31}$. Em 1911 ${ }^{32}$, Rutherford interpretou os resultados das experiências de Geiger e Marsden, propondo seu célebre modelo planetário do átomo, segundo o qual o átomo era constituído de uma parte central positivamente carregada, denominada por ele de núcleo atômico, com elétrons girando em torno dele, constituindo a eletrosfera.

Por outro lado, a radioatividade beta ( $\beta$ ), composta de elétrons ( $\mathrm{e}^{-}$) emitidos por um núcleo A que se transformava em outro núcleo $\mathrm{B}$, emissão essa conhecida como decaimento beta $(\beta)$, foi estudada pelo físico inglês Sir James Chadwick (1891-1974; PNF, 1935), em 191433, estabelecendo que aquelas partículas possuíam um espectro contínuo de energia. Registre-se que, em 191934, Rutherford voltou a realizar experiências com partículas $\alpha$, ocasião em que realizou a primeira transmutação química, ou seja, transformou o nitrogênio $\left({ }_{7} \mathrm{~N}^{14}\right)$ no oxigênio $\left.{ }_{8} \mathrm{O}^{17}\right)$ e que resultou na descoberta do próton ( $\mathrm{p}$, o núcleo do hidrogênio $-{ }_{1} \mathrm{H}^{1}$ ), com sua célebre reação nuclear: ${ }_{2} \mathrm{He}^{4}+{ }_{7} \mathrm{~N}^{14} \rightarrow{ }_{8} \mathrm{O}^{17}+{ }_{1} \mathrm{H}^{1}$. Logo em 192035 , Rutherford sugeriu que no núcleo atômico, que havia descoberto, em 1911, além de cargas positivas (p) existiam, também, cargas neutras.

Voltemos às partículas $\beta$ que, segundo Chadwick, possuíam um espectro contínuo de energia, conforme destacamos acima. Desse modo, na década de 1920, desenvolveu-se uma questão polêmica relacionada à energia dessas partículas de carga negativa. Desejava-se saber se a mesma era determinada pelas energias dos núcleos final (B) e inicial (A) ou se variava continuamente. Além do mais, havia uma questão objetiva: se um elétron é emitido pelo núcleo A que se transforma no núcleo $\mathrm{B}$ e tem energia menor que suas massas de repouso, para onde vai a energia

${ }^{30}$ Proceedings of the Royal Society of London, A82, p. 495, 1909.

${ }^{31}$ Proceedings of the Royal Society of London, A83, p. 492, 1910.

${ }^{32}$ Proceedings of the Manchester Literary and Philosophical Society, 55, p. 18, 1911; Philosophical Magazine, v. 5, p. 576, 1911; v. 21, p. 669, 1911.

${ }^{33}$ Verhandlungen der Deustschen Physikalische Gesellschaft, v. 16, p. 383, 1914.

${ }^{34}$ Philosophical Magazine, v. 37, p. 581, 1919.

${ }^{35}$ Proceedings of the Royal Society of London, A97, p. 374, 1920. 
que está faltando? Em 14 de fevereiro de 1929, o físico dinamarquês Niels Henrik David Bohr (1885-1962; PNF, 1922) escreveu uma carta para o físico inglês Sir Ralph Howard Fowler (1889-1944), na qual defendeu a tese de que o Princípio da Conservação da Energia, que parecia estar sendo violado no decaimento $\beta$, só era válido estatisticamente para fenômenos macroscópicos, sendo violado nos processos microscópicos. Aliás, essa mesma tese já havia sido defendida por Bohr, em trabalho publicado em $1924^{36}$, em parceria com os físicos, o holandês Hendrik Anthony Kramers (1894-1952) e o norte-americano John Clarke Slater (19001976), para explicar o efeito Compton (variação do comprimento de onda dos raios- $\boldsymbol{X}$ ao ser espalhado pelo elétron) descoberto, em 1923, pelo físico norteamericano Arthur Holly Compton (1892-1962; PNF, 1927) ${ }^{37}$ e, independentemente, pelo físico e químico holandês Petrus Joseph Wilhelm Debye (1884-1966; PNQ, 1936) ${ }^{38}$. É oportuno destacar que para explicar o efeito Compton, é necessário usar a conservação da energia (inclusive a de repouso einsteniana), além da conservação do momento linear ${ }^{39}$. Para resolver a polêmica referida acima, em 04 de dezembro de 1930, o físico austro-norte-americano Wolfgang Pauli Junior (1900-1958; PNF, 1945) propôs, na reunião do Group of Radioactivity of Tübingen, em uma "carta aberta" (intitulada: "To Radioactivity Ladies and Gentlemen") aos físicos, a sueco-austríaca Lise Meitner (1878-1968) e o alemão Geiger, a existência de uma partícula neutra, de massa muito pequena, não excedendo um centésimo da massa do próton, emitida junto com o elétron no decaimento $\beta$ pela "mãe" (A), cuja energia restaurava aquele princípio. Note-se que essa proposta foi também apresentada por Pauli na Reunião da Sociedade Americana de Física, realizada em junho de 1931, em Pasadena, e publicada ainda em 193140. Para uma boa discussão sobre essa polêmica, ver referência ${ }^{41}$. Registre-se que, em 1932 $2^{42}$,

${ }^{36}$ Philosophical Magazine, v. 47, p. 785; Zeitschrift für Physik 24, p. 69, 1924.

${ }^{37}$ Physical Review, v. 21, p. 207; 483; 715; 22, p. 409; Philosophical Magazine 46, p. 897, 1923.

${ }^{38}$ Physikalische Zeitschrift, v. 24, p. 161, 1923.

39 CARUSO, F.; OGURI, V. Física Moderna: Origens Clássicas e Fundamentos Quânticos. Campus/Elsevier, 2006.

40

Physical Review, 38, p. 579, 1931.

${ }^{41}$ PAIS, A. Niels Bohr's Times, in Physics, Philosophy, and Polity. Clarendon Press, 1991; BASSALO, J. M. F.; CARUSO, F. Pauli, Livraria da Física, 2013. 
Chadwick confirmou a existência do nêutron $\left({ }_{0} \mathrm{n}^{1}\right)$ como constituinte do núcleo atômico rutherfordiano, por intermédio da reação nuclear: ${ }_{2} \mathrm{He}^{4}+{ }_{5} \mathrm{~B}^{11} \rightarrow{ }_{7} \mathrm{~N}^{14}+$ ${ }_{0} \mathrm{n}^{1}$, sendo ${ }_{5} \mathrm{~B}^{11}$ um isótopo do boro.

A "partícula pauliana" foi denominada de neutrino $(v)$ (nêutron pequenino, em italiano) pelo físico ítalo-norte-americano Enrico Fermi (1901-1954; PNF, 1938), em $1934^{43}$, por ocasião em que formulou a teoria matemática do $d e-$ caimento $\beta$, segundo a qual, por intermédio de uma nova força na natureza - chamada mais tarde de força fraca - o nêutron transforma-se em um próton, com a emissão de um elétron e da "partícula pauliana", ou seja: $\mathrm{n} \rightarrow \mathrm{p}+\mathrm{e}^{-}+v$. Aliás, Fermi já havia escrito um artigo intitulado Tentativo di una Teoria della Emissione di Raggi Beta, em 193344, no qual apresentou essa mesma ideia. Contudo, esse mesmo artigo foi rejeitado pela revista Nature, para a qual ele o enviou em dezembro de 1933, sob a alegação que "ele apresentava muitas hipóteses que estavam longe de uma realidade física e, portanto, pouco interesse despertaria nos leitores". Note-se que o primeiro arranjo experimental construído com o objetivo de detectar a "partícula pauliana" foi idealizado pelos físicos norte-americanos Frederick Reines (1918-1998; PNF, 1995) e Clyde Lorrain Cowan Junior (19191974), em $1953^{45}$, ao estudarem a colisão de um fluxo de neutrinos (denotado por

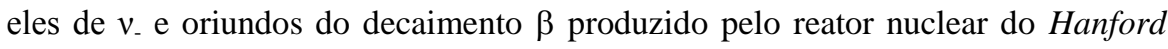
Engineering Works, situado em Washington), com prótons (p) de um cintilador líquido $\left[v_{-}+\mathrm{p} \rightarrow \mathrm{n}+\beta^{+}\left(\mathrm{e}^{+}\right)\right]$.

É interessante ressaltar que, ainda em $1953^{46}$, os físicos norte-americanos Emil John Konopinski (1911-1990) e Hormoz Massou Mahmoud (n.1918) introduziram o conceito de número leptônico $L$ e sua lei de conservação. Eles atribuíram o valor $\mathrm{L}=+1$ para o elétron (e $\mathrm{e}^{-}$) e seu neutrino associado $\left(v_{\mathrm{e}}\right)$, e $\mathrm{L}=-1$, para as suas respectivas antipartículas, o pósitron $\left(\mathrm{e}^{+}\right)$e o antineutrino associado $\left(\bar{v}_{e}\right)$. Desse modo, levando em consideração a Lei Conservação do Número Leptônico, o

${ }^{42}$ Proceedings of the Royal Society of London, A136, p. 696; 735, 1932; Nature, v. 129, p. 312, 1932.

${ }^{43}$ Nuovo Cimento, v. 11, p. 1; Zeitschrift für Physik 88, p. 161, 1934.

44

Ricerca Scientifica, v. 4, p. 491, 1933.

${ }^{45}$ Physical Review, v. 92, p. 830, 1953.

${ }^{46}$ Physical Review, v. 92, p. 1045, 1953. 
neutrino envolvido na reação de Reines-Cowan era de fato o $\bar{\nu}_{e}$. Note-se que Reines, Cowan e os físicos norte-americanos F. B. Harrison, H. W. Cruse e A. D. McGuire, usando o Savannah River Nuclear Reactor, localizado na Carolina do Sul, e um tanque de $1 / 2 \mathrm{~m}^{3}$ de água anunciaram, em $1956^{47}$ e confirmaram em 196048 , a descoberta indireta da "partícula pauliana" $\left(\bar{v}_{e}\right)$.

Vejamos agora como aconteceu a proposta da força forte. Em $1927^{49}$, o físico inglês Paul Adrien Maurice Dirac (1902-1984; PNF, 1933) publicou dois trabalhos nos quais considerou a função de onda de Schrödinger $\psi$ (e sua conjugada $\bar{\psi}$ ) como operadores [em vez de números, como o físico austríaco Erwin Schrödinger (1887-1961; PNF, 1933) havia considerado, em 192650, ao apresentar sua famosa equação: $\mathrm{H} \psi=i \hbar \partial \psi / \partial t$ ], porém sua álgebra era não-comutativa, isto é: $\psi \bar{\psi} \neq \bar{\psi} \psi$. Com esse procedimento, conhecido como Teoria Quântica da Emissão e Absorção da Radiação [também conhecida como segunda quantização, que considera os operadores criação $\left(\mathrm{a}^{+}\right)$, destruição $\left(\mathrm{a}^{-}\right)$e número de ocupação $\left(\mathrm{N}=\mathrm{a}^{+} \mathrm{a}^{-}\right)$], Dirac quantizou o campo eletromagnético, procedimento esse que deu origem ao desenvolvimento da Eletrodinâmica Quântica (Quantum Electrodynamics - QED).

Segundo esta teoria, o elétron é preso ao próton, no caso do átomo de hidrogênio $(\mathrm{H})$, em virtude da troca de "fótons virtuais". Como essas partículas eletrizadas estão sob a ação da força eletromagnética, a "segunda quantização diraciana" vista acima, significa dizer que os fótons [nome cunhado, em 1926 ${ }^{51}$, pelo químico norte-americano Gilbert Newton Lewis (1875-1946) e hoje denotado por $\gamma]$ são as partículas mediadoras ("quanta") da força (interação) eletromagnética.

Por outro lado, a descoberta do nêutron por Chadwick, em 1932, como uma das partículas constituintes do núcleo atômico rutherfordiano, juntamente com o próton, provocou outra grande dificuldade para os físicos, qual seja, a de explicar a razão dos prótons não se repelirem pela força coulombiana (eletro-

${ }^{47}$ Science, v. 124, p. 103, 1956.

${ }^{48}$ Physical Review, v. 117, p. 159, 1960.

${ }^{49}$ Proceedings of the Royal Society, A114, p. 243; 710, 1927.

${ }^{50}$ Annales de Physique Leipzig, v. 79, p. 361; 489; 734; 747; v. 80, p. 437; v. 81, p. 136, 1926.

${ }^{51}$ Nature, v. 118, p. 874, 1926.

Bassalo, J. M. F. 
magnética) no interior do núcleo. Para resolver essa dificuldade, ainda em 1932, os físicos, o alemão Werner Karl Heisenberg (1901-1976; PNF, 1932) ${ }^{52}$, o russo Dimitrij Iwanenko (1904-1994) ${ }^{53}$ e o italiano Ettore Majorana (1906-1938) propuseram a hipótese de que os prótons e os nêutrons, enquanto partículas constituintes do núcleo atômico se comportavam como partículas únicas - os núcleons que interagiam por intermédio de uma força atrativa capaz de superar a "repulsão coulombiana".

Ainda em $1932^{54}$, Heisenberg defendeu a ideia de que os núcleons deveriam se caracterizar por um novo número quântico, o hoje conhecido spin isotópico ou isospin (I). Segundo nos fala o romancista italiano Leonardo Sciascia (19211989), em seu livro Majorana Desapareceu (Rocco, 1991), Majorana formulou a Teoria dos Núcleons seis meses antes de Heisenberg. Depois de apresentá-la aos seus colegas do Instituto de Física da Universidade de Roma, se recusou a publicála, bem como proibiu que seu colega Fermi o apresentasse no Congresso de Física que iria ser realizado em Paris ${ }^{55}$.

A ideia da energia de ligação entre núcleons ainda foi tratada por Heisenberg, em 193356, ao mostrar que essa energia aumentava de uma maneira aproximadamente igual ao número de núcleons. Em vista disso, afirmou que a partícula $\alpha$ apresentava uma estrutura de saturação dessa energia. Essa ideia foi logo contestada por Majorana em trabalho publicado ainda em 193357, ao afirmar que era o dêuteron (núcleo do hidrogênio pesado: ${ }_{1} \mathrm{H}^{2}={ }_{1} \mathrm{D}^{2}$ ) e não a partícula $\alpha$ que era completamente saturada pela "força de Heisenberg". Observe-se que o ${ }_{1} \mathrm{D}^{2}$ foi descoberto, em $1932^{58}$, pelos químicos norte-americanos Harold Clayton Urey (1893-1981; PNQ, 1934), Ferdinand Graft Brickwedde (1903-1989) e George Moseley Murphy (1903-1969) ${ }^{59}$.

${ }^{52}$ Zeitschrift für Physik, v. 77, p. 1, 1932.

${ }^{53}$ Nature, v. 129, p. 798, 1932.

${ }^{54}$ Zeitschrift für Physik, v. 78, p. 156, 1932.

${ }^{55}$ BASSALO, J. M. F.; CARUSO, F. Fermi. Livraria da Física, 2013.

${ }^{56}$ Zeitschrift für Physik, v. 80, p. 587, 1933.

${ }^{57}$ Zeitschrift für Physik, v. 82, p. 137; Ricerca Scientifica, v. 4, p. 559, 1933.

${ }^{58}$ Physical Review, v. 39, p. 164; 864; 40, p. 1, 1932.

${ }^{59}$ BASSALO, J. M. F.; CARUSO, F Heisenberg. Em preparação. 
A polêmica da "força de Heisenberg" foi finalmente resolvida, em $1935^{60}$, quando o físico japonês Hideaki Yukawa (1907-1981; PNF, 1949) propôs que aquela "força" decorria da troca entre eles da partícula $\mathrm{U}$ (como a denominou Yukawa), porém sua massa deveria ser $\mathrm{m}_{\mathrm{U}}=200 \mathrm{~m}_{\mathrm{e}}$ (sendo $\mathrm{m}_{\mathrm{e}}$ a massa do elétron). Para chegar a esse valor, Yukawa admitiu que a energia potencial $V$ de dois núcleons em repouso seria dada por: $\mathrm{V}=-(\mathrm{A} / \mathrm{r}) \exp (-\mu \mathrm{r})$, em que A uma é constante $\mathrm{e} \mu=\mathrm{m}_{\mathrm{U}} \mathrm{c} / \hbar \approx 10^{13} \mathrm{~cm}^{-1}$. Portanto, para Yukawa a força nuclear [mais tarde chamada de força (interação) forte] era de curto alcance e mediada (em analogia com a troca de fótons entre elétron e próton no átomo de $\mathrm{H}$, conforme visto acima) por uma partícula de massa intermediária entre a massa do elétron $\left(\mathrm{m}_{\mathrm{e}}\right)$ e a massa do próton $\left(\mathrm{m}_{\mathrm{p}}\right)$, razão pela qual a mesma ficou conhecida, inicialmente, como yukon, mesotron e, hoje, méson pi $(\pi)$.

É oportuno registrar que a existência dessa partícula foi confirmada nas experiências realizadas, em $1947^{61}$, pelos físicos, o brasileiro Cesare (César) Mansueto Giulio Lattes (1924-2005), os ingleses Hugh Muirhead (1925-2007) e Sir Cecil Frank Powell (1903-1969; PNF, 1950), e o italiano Guiseppe Paolo Stanislao Occhialini (1907-1993), nas quais observaram que a incidência de raios cósmicos em emulsões nucleares colocadas nos Alpes (Suíça) e em Chacaltaya (Bolívia) produzia dois tipos de mésons: primários (hoje, múons - $\mu$ ) e secundários (hoje, píons - $\pi$ ).

Assim, a descoberta de mais duas forças (interações) na Natureza elevava para quatro o número das forças naturais: gravitacional, eletromagnética, fraca e forte. Tais forças, no entanto, são bem distintas, pois suas constantes de acoplamento, em valores aproximados, valem, respectivamente: $10^{-39}, 10^{-2}, 10^{-10}$ e 10 .

Durante mais de 20 anos, essas quatro forças (interações) permaneceram independentes, pois, conforme vimos anteriormente, a tentativa de unificar (via geometrização) as duas primeiras foi malograda. Diferentemente dessa via geométrica, a tentativa de unificar aquelas forças começou a ser viabilizada graças ao desenvolvimento das Teorias de "Gauge" ("Calibre"). Vejamos como isso ocorreu. Em 195462, os físicos, o sino-norte-americano Chen Ning Yang (n.1925; PNF, 1957) e o norte-americano Robert Laurence Mills (n.1927), propuseram uma Teoria de "Gauge" não-Abeliana para estudar a interação forte. Registre-se que, em

${ }^{60}$ Proceedings of the Physical Mathematics Society of Japan, v. 17, p. 48, 1935.

${ }^{61}$ Nature, v. 159, p. 694, 1947.

${ }^{62}$ Physical Review, v. 96, p. 191, 1954.

Bassalo, J. M. F. 
1955, o físico inglês Ronald Shaw (n.1929) defendeu sua Tese de Doutoramento, sob a orientação do físico paquistanês Abdus Salam (1926-1996; PNF, 1979), na qual havia uma proposta semelhante à de Yang-Mills. No entanto, por não ser renormalizável para bósons (partículas que têm spin zero ou inteiro) massivos, essa Teoria de Yang-Mills-Shaw (TYMS) não poderia descrever as interações fracas, já que essas são mediadas por partículas massivas, conforme a proposta apresentada por Klein, em $1938^{63}$. Segundo essa proposta, o decaimento $\beta$ seria mediado por bósons vetoriais $($ spin $=1$ ) massivos e carregados, aos quais denominou de $\omega$ (hoje, W). Assim, para Klein, esse decaimento seria dado por (em notação da época): $\mathrm{n} \rightarrow \mathrm{p}+\omega^{-} \rightarrow \mathrm{e}^{-}+\mathrm{v}$.

A ideia de as interações fracas serem mediadas por bósons vetoriais aventadas por Klein, conforme vimos acima, foi retomada, em $1957^{64}$, pelo físico norte-americano Julian Seymour Schwinger (1918-1994; PNF, 1965) e, em $1958^{65}$, pelos físicos norte-americanos Richard Phillips Feynman (1918-1988; PNF, 1965) e Murray Gell-Mann (n.1929; PNF, 1969), em sua proposta da famosa Teoria V-A, que universalizou a interação fraca, e segundo a qual esse tipo de interação poderia ser devido à troca dos bósons kleinianos. Estimulado pela leitura do trabalho de Feynman-Gell-Mann, o físico brasileiro José Leite Lopes (1918-2006), ainda em $1958^{66}$, publicou um artigo no qual considerou que a constante de acoplamento da interação eletromagnética (constante de estrutura fina $\alpha \approx 1 / 137 \approx 10^{-2}$ ) com a matéria seria igual à constante de acoplamento da interação fraca $\left(\mathrm{G}_{\mathrm{W}}\right)$ também com a matéria, isto é: $\alpha=G_{\mathrm{W}}$. Desse modo, Leite Lopes propôs que a interação elétron-nêutron só poderia ser realizada por intermédio de um bóson vetorial neutro, o hoje conhecido $Z^{0}$, chegando a estimar a sua massa em cerca de 60 massas do próton $\left(60 \mathrm{~m}_{\mathrm{p}}\right)$. Observe-se que trabalhos semelhantes a esses relacionados com a unificação das interações fraca e eletromagnética foram realizados, ainda em 1958, pelos físicos norte-americanos Sidney Arnold Bludman (n.1927) ${ }^{67}$ e Gerald Feinberg (1933-1992)68; em 1959, por Salam e o físico inglês John Clive Ward

\footnotetext{
63
}

Journal de Physique et le Radium, v. 9, p. 1, 1938.

64

Annals of Physics NY 2, p. 407, 1957.

65

Physical Review, v. 109, p.109, 1958.

66

Nuclear Physics, v. 8, p. 234, 1958.

67

Nuovo Cimento, v. 9, p. 433, 1958.

68

Physical Review, v. 110, p. 1482, 1958. 
(1924-2000) ${ }^{69}$, e pelo físico norte-americano Sheldon Lee Glashow (n.1932; PNF, $1979)^{70}$ (este trabalho fez parte de sua Tese de Doutoramento, orientada por Schwinger e defendida em 1958); e Gell-Mann ${ }^{71}$; e, em $1960^{72}$, pelos físicos sinonorte-americanos Tsung-Dao Lee (n.1926; PNF, 1957) e Yang.

A TYMS voltou a ser objeto de pesquisa por parte do físico japonês Yoichiro Nambu (n.1921; PNF, 2008) ao descobrir, em $1960^{73}$, a quebra de espontânea de simetria nessa teoria, usando uma analogia com a supercondutividade [descoberta em $1911^{74}$, pelo físico holandês Heike Kamerlingh Onnes (1853-1926; PNF, 1913)]. Esta descoberta foi confirmada, em 1961, pelo físico inglês Jeffrey Goldstone (n. 1933) 75 e, também, por Nambu e G. Jona-Lasínio ${ }^{76}$. Esses trabalhos mostravam que essa quebra de simetria era acompanhada de partículas nãomassivas, logo denominadas de bósons de Nambu-Goldstone (bN-G) ou, simplesmente, bósons de Goldstone (bG), também conhecido como Teorema de Goldstone (TG). Logo em seguida, em 196277, Goldstone e os físicos, o paquistanês Abdus Salam (1926-1996; PNF, 1979) e o norte-americano Steven Weinberg (n.1933; PNF, 1979) mostraram que a existência desses bósons era um resultado geral da Teoria Quântica de Campos. Em 1964, em trabalhos independentes, Higgs $^{78}$, Englert e Brout ${ }^{79}$, e Guralnik, Hagen e Kibble ${ }^{80}$, encontraram um meca-

69

Nuovo Cimento, v. 11, p. 568, 1958.

70

Nuclear Physics, v. 10, p. 107, 1959.

${ }^{71}$ Review of Modern Physics, v. 31, p. 834, 1959.

${ }^{72}$ Physical Review, v. 119, p. 1410, 1960.

${ }^{73}$ Physical Review Letters, v. 4, p. 382, 1960.

${ }^{74}$ Communications from the Physical Laboratory of Leiden 122B e 124C, 1911.

${ }^{75}$ Nuovo Cimento, v. 19, 154, 1961.

76

Physical Review, v. 122, p. 345; 124, p. 246, 1961.

${ }^{77}$ Physical Review, v. 127, p. 965, 1962.

${ }^{78}$ Physics Letters, v. 12, p. 132; Physical Review Letters, v. 13, p. 508, 1964.

79

Physical Review Letters, v. 13, p. 321, 1964.

80

Physical Review Letters, v. 13, p. 585, 1964.

Bassalo, J. M. F. 
nismo que tornava massivos os bN-G. Esse mecanismo ficou conhecido como mecanismo de Higgs, e o bóson de spin nulo correspondente de tal mecanismo, como bóson de Higgs (H), cuja massa foi estimada em experiências posteriores, porém com um grande intervalo, até finalmente ser detectada, em 2012, como veremos mais adiante.

Registre-se que, em $1966^{81}$, o físico brasileiro Jorge André Swieca (19361980) (de origem polonesa), com a participação de David Kastler e Derek W. Robinson, apresentou uma demonstração axiomática do TG, com as seguintes hipóteses básicas: 1) invariância de Lorentz; 2) espaço de Hilbert com norma positiva definida; 3 ) simetria global.

Sobre os trabalhos de Higgs descritos acima, há um aspecto curioso a registrar. No primeiro trabalho ( $P L$ 12:132), ele mostrou que as partículas sem massa previstas por Goldstone não ocorrem quando a simetria local é espontaneamente quebrada na Teoria de Campo Relativística. Em vista disso, ele preparou um segundo artigo no qual apresentou o seu famoso mecanismo de Higgs e o enviou para a Physics Letters (PL) que foi rejeitado pelos editores com o argumento de que "o artigo não era relativamente óbvio para a física". Então, Higgs acrescentou um parágrafo extra e o enviou para a Physical Review Letters (PRL) que o publicou, ainda em $1964^{82}$, mas posteriormente ao artigo de Englert e Brout ${ }^{83}$. Note-se que, em sua Nobel Lecture (NL), Higgs reproduz esse fato curioso sobre a rejeição de seu artigo pela PL, que o havia recebido no dia 31 de julho de 1964. O editor, que trabalhava no CERN, em sua rejeição sugeriu que Higgs desenvolvesse mais suas ideias e o enviasse para a Il Nuovo Cimento. Higgs ficou indignado, pois achava que o referee ("árbitro") não havia percebido o ponto principal de seu artigo e, desse modo, preparou um novo artigo com uma observação final: "É importante destacar um aspecto essencial desse tipo de teoria que é a previsão de multipletos incompletos de bósons escalares e vetoriais" - e o enviou para a PRL, na qual chegou no dia 31 de agosto. Ainda em sua NL, Higgs comenta que só tomou conhecimento do trabalho de Englert e Brout por intermédio de um pedido feito pelo referee de seu trabalho, ou seja, o que era de comentar a relação entre o seu trabalho e o dos físicos belgas. Somente depois de vinte anos, em 1984, em uma Confe-

\footnotetext{
${ }^{81}$ Communications in Mathematical Physics, v. 2, p. 108, 1966.

82

Physical Review Letters, v. 13, p. 508, 1964.

83

Physical Review Letters, v. 13, p. 321, 1964 (Recebido no dia 22 de junho).
} 
rência Internacional, Nambu disse-lhe que havia sido o referee dos dois famosos artigos.

A unificação das forças eletromagnética e fraca especulada nos trabalhos referidos acima foi finalmente formalizada nos artigos do físico norte-americano Steven Weinberg (n.1933; PNF, 1979), em $1967^{84}$ e de Salam, em $1968^{85}$, a conhecida Teoria Eletrofraca. Segundo essa teoria, baseada no grupo $\mathrm{SU}(2) \otimes \mathrm{U}(1)$, a força eletrofraca é mediada por quatro quanta: o fóton $(\gamma)$, partícula não-massiva e mediadora da interação eletromagnética e os bósons vetoriais $\left(\mathrm{W}^{ \pm}, \mathrm{Z}^{0}\right.$ ) (a notação de $\mathrm{Z}^{0}$ foi sugerida por Weinberg), de massas respectivas: $\approx 87 \mathrm{~m}_{\mathrm{p}} \mathrm{e} \approx 97 \mathrm{~m}_{\mathrm{p}}$.

Observe-se que nessa Teoria de Salam-Weinberg (TSW), as constantes de acoplamento das interações, a eletromagnética $(\alpha \approx 1 / 137)$ e a fraca $\left(G_{W}\right)$ são relacionadas pela expressão: $\alpha=\mathrm{G}_{\mathrm{W}} \operatorname{sen}\left(\theta_{\mathrm{W}}\right)$, onde $\theta_{\mathrm{W}}$ é o ângulo de Weinberg. E mais ainda, nessa TSW, inicialmente as partículas $\mathrm{W}^{ \pm}, \mathrm{Z}^{o}, \gamma$ têm massa nula e estão sujeitas à simetria "gauge". No entanto, por intermédio do mecanismo de Higgs, do qual participam o dubleto Higgs $\left(\mathrm{H}^{+}, \mathrm{H}^{0}\right)$ e seu antidubleto $\left(\mathrm{H}^{-}, \overline{\mathrm{H}}^{0}\right)$, há a quebra espontânea dessa simetria, ocasião em que o fóton $(\gamma)$ permanece com massa nula, porém os $\mathrm{W}^{ \pm}$adquirem massas por incorporação dos bósons carregados $\left(\mathrm{H}^{ \pm}\right)$, ao passo que $\mathrm{Z}^{0}$ adquire massa de uma parte dos bósons neutros $\left(\mathrm{H}^{0}+\overline{\mathrm{H}}^{0}\right)$, ficando a outra parte $\left(\mathrm{H}^{0}-\overline{\mathrm{H}}^{0}\right)$ como uma nova partícula bosônica escalar (spin 0), o que hoje é conhecido como bóson de Higgs $(\mathrm{H})^{86}$. Registre-se que uma simetria de um sistema é dita quebrada espontaneamente se o mais baixo estado do sistema é não-invariante sob as operações dessa simetria ${ }^{87}$.

Por outro lado, a TSW apresentava uma grande dificuldade, pois ela não era renormalizável, ou seja, apareciam divergências (infinitos) nos cálculos envolvendo os quatros quanta, característicos dessa teoria. Para contornar essa dificuldade, em 197188, Glashow e o físico grego-norte-americano John Iliopoulos (n.1940) examinaram o cancelamento (renormalizabilidade) na TYMS. Ainda em

${ }^{84}$ Physical Review Letters, v. 19, p. 1264, 1967.

85

Proceedings of the Eighth Nobel Symposium, p. 367, 1968.

86

Salam, 1991, op. cit.

${ }^{87}$ CATTANI, M.; BASSALO, J. M. F. Higgs Field, Higgs Mechanism and the Boson of Higgs. Disponível em: 〈http://publica-sbi.if.usp.br/PDFs/pd1670.pdf〉.

${ }^{88}$ Physical Review D3, p. 1043, 1971.

Bassalo, J. M. F. 
1971, o físico holandês Gerardus 't Hooft (n.1946; PNF, 1999) publicou dois artigos nos quais estudou aquele cancelamento. No primeiro ${ }^{89}$, ele usou bósons vetoriais não-massivos e não considerou o mecanismo de quebra espontânea de simetria de Guralnik-Hagen-Kibble-Brout-Englert-Higgs; e, no segundo ${ }^{90}$, ele trabalhou com partículas massivas e o mecanismo referido acima. Por outro lado, o físico holandês Martinus Justinus Godefridus Veltman (n.1931; PNF, 1999), que havia sido orientador da Tese de Doutoramento de 't Hooft, observou que o modelo de regularização dimensional de 't Hooft só eliminava os infinitos dos diagramas de Feynman de dois laços. Porém, para mais de dois laços, os infinitos permaneciam. Assim, em 197291, 't Hooft e Veltman desenvolveram o modelo de regularização dimensional contínua que conseguia eliminar todos os infinitos dos diagramas de Feynman. É oportuno destacar que, ainda em 1972, os físicos argentinos Juan José Giambiagi (1924-1996) e Carlos Guido Bollini (1926-2009)92 e o koreano-norte-americano Benjamin W. Lee (1935-1977) ${ }^{93}$, desenvolveram o mesmo tipo de regularização. Desse modo, os trabalhos de 't Hooft, Veltman, Giambiagi, Bollini e Lee conseguiram resolver a grande dificuldade da TSW, ou seja, a sua renormalização. Estava assim completada a teoria da força (interação) eletrofraca. Só faltava a detecção de suas partículas mediadoras: $\mathrm{W}^{ \pm}$e $\mathrm{Z}^{0} 94$.

Note-se que a renormalização [termo cunhado pelo físico norteamericano Robert Serber (1909-1997), em 1936] ${ }^{95}$ é um método pelo qual os infinitos de uma Teoria de Campo (TC) são absorvidos em seus parâmetros livres, de modo que resultam valores finitos nos cálculos, em todas as ordens de perturbação, para todos os observáveis envolvidos nos fenômenos físicos tratados pela TC.

É oportuno destacar que a importância da descoberta do bóson de Higgs decorre do fato de que o Standard Model (SM) ("Modelo Padrão") da Física das Partículas Elementares, modelo este composto pela TSW e pela Cromodinâmica

\footnotetext{
${ }^{89}$ Nuclear Physics B33, p. 173, 1971.

${ }^{90}$ Nuclear Physics B35, p. 167, 1971.

91

Nuclear Physics B44; B50, p. 189; 318, 1972.

92 Nuovo Cimento B12, p. 20; Physics Letters B40, p. 566, 1972.

${ }^{93}$ Physical Review D5, p. 823, 1972.

${ }^{94}$ Para detalhes dos trabalhos de 't Hooft e Veltman, ver: VELTMAN, M. Facts and Mysteries in Elementary Particles, World Scientific, 2003.

${ }^{95}$ Physical Review, v. 49, p. 545, 1936.
} 
Quântica (Quantum Cromodynamics - QCD) [que explica a força forte, mediada pelo glúon (g), que liga os quarks (q)], a massa das partículas é um parâmetro, ou seja, ela é dada em função da massa do elétron $\left(\mathrm{m}_{\mathrm{e}}\right)$ cujo valor é determinado experimentalmente, valor esse que decorre das experiências de J. J. Thomson, em $1897^{96}$, que calculou a relação $\mathrm{e} / \mathrm{m}_{\mathrm{e}}$, e os experimentos do físico norte-americano Robert Andrews Millikan (1868-1953; PNF, 1923), para determinar a carga do elétron (e), a partir de 1906 e definida em 191297.

Concluindo este artigo, é oportuno fazer três comentários sobre o H. Primeiro, sobre o nome em que ele é conhecido: partícula Deus em virtude do título do livro escrito pelo físico norte-americano Leon Max Lederman (n. 1922; PNF, 1988) em parceria com o escritor norte-americano Dick Teresi: The God Particle: If the Universe Is the Answer, What Is the Question? (Delta Book, 1994), no qual há uma descrição histórico-conceitual da Física das Partículas Elementares e, portanto, envolvendo essa "misteriosa" partícula que é a responsável pela massa das partículas, segundo vimos acima. Aliás, sobre o título principal desse livro, há o seguinte esclarecimento em sua página 22: "Why God Particle? Two reasons. One, the publisher wouldn't let us call it the Goddamn Particle, though that might be a more appropriate title, given its villainous nature and the expense it is causing. And two, there is a connection of sorts, to another book, a much older one..." ("Por que Partícula Deus? Duas razões. Uma, o editor não permitiu que eu a chamasse de Partícula Maldita, embora este possa ser um título apropriado, o mesmo supõe sua natureza infame e causa prejuízo. Dois, existe uma conexão natural a outro livro, e muito mais velho..."). É oportuno dizer que Lederman se refere a Bíblia pois, em seguida a essa afirmação, ele cita uma passagem do Gênesis 11:19. É interessante ressaltar que Salam (1991, op. cit.), diz que a partícula $Z^{0}$ é a verdadeira partícula de Deus, pois ela é a partícula que faz o elétron atômico ter uma órbita espiralada e, portanto, o átomo torna-se quiral (a imagem especular é diferente da imagem real). Essa é a propriedade que faz com a maioria dos 20 aminoácidos (responsáveis pela vida) serem moléculas quirais. Desse modo, considerando que a Química da Vida se deve à essa quiralidade, Salam (op. cit.) escreveu a seguinte frase: "Existe uma certeza cada vez maior de que a força eletrofraca é a verdadeira força da vida e que DEUS criou a partícula $Z^{0}$ para fornecer a quiralidade às moléculas da vida". Assim, creio que podemos dizer que $Z^{0}$ é a verdadeira Partícula de Deus.

${ }^{96}$ Philosophical Magazine, v. 44, p. 295, 1897.

${ }^{97}$ Transactions of the American Electrochemical Society, v. 21, p. 185, 1912. 
O segundo comentário relaciona-se com a sua detecção. Proposto o $\mathrm{H}$, como vimos acima, os grandes aceleradores de partículas, principalmente, o $\boldsymbol{L E P}$ (Large Electron-Positron Collider) Working Group for Higgs Boson Searches do EP/CERN [Colaborações: Apparatus for LEP PHysics (ALEPH), Detector with Lepton, Photon and Hadron Identification (DELPHI), Terceiro Experimento do LEP (L3), Omni-Purpose Apparatus for LEP) (OPAL)], e os anéis de colisão próton-antipróton [ $p \bar{p}$ (ppbar)] do FERMILAB [Tevatron New Phenomena \& Higgs Working Group: Combined D0/CDF (TEVNPHWG:D0/CDF)] e o LHC/CERN (Colaborações: ATLAS e CMS) começaram a busca por sua confirmação. Note-se que o LEP é um anel de $27 \mathrm{~km}$ e energia inicial de $91 \mathrm{GeV}$, quando construído em 1989; o Tevatron é um anel de 6,3 km e energia de $2 \mathrm{TeV}$, construído em 1983 e desativado em 30 de setembro de 2011; e o LHC (sucessor do LEP, a partir de 2000), mantendo $27 \mathrm{~km}$ e energia de $7 \mathrm{TeV}$ (em 2011) e $8 \mathrm{TeV}$ (em 2012), por feixe. (Note que: $1 \mathrm{GeV}=10^{9} \mathrm{eV}$ e que $1 \mathrm{TeV}=10^{12} \mathrm{eV}$; sendo 1 $\mathrm{eV}=1,602176462 \times 10^{-19} \mathrm{~J} \mathrm{e} \mathrm{E}=\mathrm{mc}^{2}=\mathrm{m}$, considerando $\left.\mathrm{c}=1\right)$.

A busca de $\mathrm{H}$ começou nas duas últimas décadas do Século 20 e prolongou-se até 2012, com várias evidências de sua existência encontradas pelas colaborações do LEP: ALEPH, DELPHI, L3, OPAL, TEVNPHWG:D0/CDF (sendo que dessas colaborações do LEP, nenhuma evidência foi confirmada e indicavam uma massa para o $\mathrm{H}$ de $115 \mathrm{GeV}$, menor do que o valor realmente detectado, como veremos a seguir), e pelo ATLAS e CMS, conforme se pode ver nos artigos de Gregorio Bernardi, Marcela Carena (física argentina nascida em 1962) e Thomas R. Junk, Higgs Bosons Theory and Searches ${ }^{98}$.

Contudo, a sua existência só foi considerada dentro dos padrões internacionais (com nível de precisão de $5 \sigma$, ou seja, menos do que um em um milhão de chances de erro) em 2012, quando a colaboração TEVNPHWG:D0/CDF (ar$X i v: 1207.0449 v 1$ [hep-ex], 02 de julho) anunciou (depois de uma informação extra-oficial do LHC) a existência de $\mathrm{H}$ com $\mathrm{m}_{\mathrm{H}} \approx(114-130) \mathrm{GeV}$, com um nível de precisão de apenas $3 \sigma$, e as colaborações $\operatorname{ATLAS}^{99}$ e $\mathrm{CMS}^{100}$ anunciaram, por sua vez, em 04 de julho, terem realmente detectado (com $5 \sigma$ ) o $\mathrm{H}$ com os respectivos valores de $\mathrm{m}_{\mathrm{H}} \approx 126,5 \mathrm{GeV}$ e 125, $3 \pm 0,6 \mathrm{GeV}$, após observaram, basicamente, o

${ }^{98}$ Physical Review D86, article number 010001 (2012)] e DELLA NEGRA, M.; JENNI, P.; VIRDEE, T. S. Journey in the Search for the Higgs Boson: The ATLAS and CMS Experiments at the Large Hadron Collider (Science, v. 338, p. 1560, 21 dez. de 2012.)

99

Physics Letters, B716, p. 1, 2012.

100

Physics Letters, B716, p. 30, 2012. 
decaimento de $\mathrm{H}$ nos canais: $\mathrm{H} \rightarrow 2 \mathrm{Z}^{0}$ e $\mathrm{H} \rightarrow 2 \gamma$. Note-se que os demais canais observados pelo LHC: $\mathrm{H} \rightarrow b \bar{b}$ (bbar) não apresentava nenhuma evidência de $\mathrm{H}, \mathrm{o}$ que levou alguns físicos teóricos a especular que essa partícula era "fermiofóbica", e o canal $\mathrm{H} \rightarrow \mathrm{W}^{+}+\mathrm{W}^{-}$também observado pelo LHC.

Note-se que, o canal $\mathrm{H} \rightarrow b \bar{b}$ era o candidato mais provável, e no final a descoberta aconteceu no canal $\mathrm{H} \rightarrow 2 \gamma$ (e $2 \mathrm{Z}^{0}$ ), não porque era mais abundante, mas sim por ser o mais limpo, e com maior capacidade de rejeição do background hadrônico (José Guilherme Rocha de Lima, por e-mail, em 22/11/2013; Gilvan Augusto Alves, por e-mail, em 30/11/2013).

É oportuno registrar que nessas duas colaborações trabalharam físicos brasileiros. Na ATLAS, dentre os quase três mil cientistas que participaram dessa colaboração, estiveram presente treze (13) cientistas brasileiros (físicos e engenheiros), assim distribuídos: José Guilherme Rocha de Lima (n.1967) do Fermi National Laboratory, Denis Oliveira Damazio (n.1975), do Physics Department do Brookhaven National Laboratory (USA), e os das seguintes instituições brasileiras: 1) Instituto de Física da Universidade Federal do Rio de Janeiro e Instituto Alberto Luiz Coimbra de Pós-Graduação em Engenharia (IF/UFRJ/COPPE) - Luiz Pereira Calôba (n.1944), Carmen (Lucia Lodi) Maidantchik (n.1967), Fernando Marroquim (Leão de Almeida Junior) (n.1948), Andre Asevedo Nepomuceno (n.1979), Marcelo Perantoni (n.1973) e José Manoel de Seixas (n.1954); 2) Faculdade de Engenharia Elétrica da Universidade Federal de Juiz de Fora (UFJF) Augusto Santiago Cerqueira (n.1974) e Luciano Manhães de Andrade Filho (n.1978); 3) Departamento de Ciências Naturais da Universidade Federal de São João del Rei (DCN/UFSJ) - Maria Aline Barros do Vale (n.1961); 4) Instituto de Física da Universidade de São Paulo (IF/USP) - Marisilvia Donadelli (n.1968) e Marco Aurélio Lisboa Leite (n.1966). Na CMS, com também quase três mil cientistas, estiveram presente trinta e cinco (35) brasileiros de três instituições brasileiras e assim distribuídos: 1) Centro Brasileiro de Pesquisas Físicas (CBPF) - Gilvan Augusto Alves (n.1960), Lucas (de Brito) Cavalcanti (n.1986), Marcos Correa Martins Junior (n.1984), Thiago (dos Reis) Martins (n.1984), Maria Elena Pol (n.1948) e Moacyr Henrique Gomes e Souza (n.1944); 2) Instituto de Física da Universidade Estadual do Rio de Janeiro (IF/UERJ) - Walter Luiz Alda Júnior (n.1984), Wagner (de Paula) Carvalho (n.1968), Analu Custódio (n.1987), Eliza Melo da Costa (n.1981), Dilson de Jesus Damião (n.1979), Carley (Pedro) de Oliveira Martins (n.1951), Sandro Fonseca de Souza (n.1975), Helena (Brandão) Malbouisson (n.1978), Magdalena Malek (n.1981), Diego Matos Figueiredo (n.1984), Luiz (Martins) Mundim (Filho) (n.1966), Hélio Nogima (n.1963), Vitor 
Oguri (n.1951), Wanda Lúcia Prado da Silva (n.1953), Alberto (Franco de Sá) Santoro (n.1941), André Sznajder (n.1967) e Antônio Vilela Pereira (n.1982); 3) Instituto de Física Teórica da Universidade Estadual Paulista (IFT/UNESP) Tiago Santos dos Anjos (n.1986), César Augusto Bernardes (n.1985), Flávia de Almeida Dias (n.1987), Thiago Rafael Fernandez Perez Tomei (n.1983), Eduardo de Moraes Gregores (n.1958), Rogério Luiz Iope (n.1965), Caio Lagana Fernandes (n.1987), Sérgio Morais Lietti (n.1970), Franciole da Cunha Marinho (n.1981), Pedro Galli Mercadante (n.1969), Sérgio Ferraz Novaes (n.1956) e Sandra dos Santos Padula (n.1956).

Vejamos o terceiro comentário sobre o $\mathrm{H}$, agora relacionado com a sua massa $\left(\mathrm{m}_{\mathrm{H}}\right)$, que apresenta algumas dificuldades. Por exemplo, embora o $\mathrm{H}$ e todas as partículas do SM entrem em correções radiativas (loops) para a massa bare ("nua") do $\mathrm{H}$, o problema que surge é o de evitar que essas correções cresçam indefinidamente fazendo com que $\mathrm{m}_{\mathrm{H}}>1 \mathrm{TeV}$ (Alves, e-mail citado). É oportuno esclarecer que o astrofísico brasileiro Mario Novello (n.1942), em artigos publicados em $2010^{101}$, em $2011^{102}$, e em $2012^{103}$ [este, em parceria com o físico brasileiro Eduardo Henrique Silva Bittencourt (n.1985)], propõe o mecanismo gravitacional (a ação gravitacional do Universo sobre suas partes) como gerador da massa de todas as partículas elementares, inclusive à do bóson de Englert-Higgs (EH), como Novello o denomina ${ }^{104}$. Outro problema é que a $\mathrm{m}_{\mathrm{H}}$ não explica as massas das partículas que decorrem da Teoria da Supersimetria. Vejamos como esta foi formulada. Em 1971 105 , os físicos, o francês Jean Loup Gervais (n.1936) e o japonês Bunji Sakita (n.1930), descobriram uma nova simetria - logo conhecida como supersimetria (SUSY - "supersymmetry") [que envolve energias da ordem de $10^{20}$ $\mathrm{GeV}$ (ou dimensões da ordem de $10^{-34} \mathrm{~cm}$ )] - que converte bósons em férmions.

${ }^{101}$ arXiv:1003.5126v2 [physics.gen-ph] 5 de abril de 2010.

${ }^{102}$ Classical and Quantum Gravity, v. 28, a.n. 035003, 2011; International Journal of Modern Physics A26, p. 3781, 2011.

103

Physical Review D86, p. a.n. 063510, 2012.

104

ELBAZ, E. O que, afinal, confere massa ao bóson de Higgs? Ciência Hoje, v. 139, p. 70, dez. 2013.

105

Nuclear Physics B34, p. 632, 1971. 
Em 1973, trabalhos independentes dos físicos russos D. V. Volkov e V. P. Akulov ${ }^{106}$ e de Gervais e Sakita ${ }^{107}$, desenvolveram a hoje conhecida Teoria da Supersimetria (TSS). Segundo essa teoria, cada partícula deverá possuir uma supercompanheira (partículas $\boldsymbol{S U S Y}$ ou ino) com propriedades idênticas, exceto no valor de seu spin, que vale o spin da partícula correspondente, subtraído de 1/2. Assim, para as partículas mediadoras das interações (gravitacional: gráviton, de spin 2; forte: glúon, de spin 1; fraca: $\mathrm{W}^{ \pm}$e $\mathrm{Z}^{0}$, de spin 1; eletromagnética: fóton, de spin 1) suas supercompanheiras (partículas ino) são, respectivamente: gravitino, de spin $3 / 2$; gluino, de $\operatorname{spin} 1 / 2$; wino $^{ \pm}$, de $\operatorname{spin} 1 \frac{1}{2}$; zino $^{\boldsymbol{0}}$, de spin $1 / 2$; e fotino, de spin $1 / 2$. Para as partículas constituintes da matéria (quarks e léptons, ambas de spin 1/2), suas supercompanheiras são: squarks e sléptons, de spin 0.

Com a previsão da H, em 1964, como responsável pela massa das partículas elementares, como registramos acima, surgiu a necessidade de encontrar uma versão do SM para incorporar a SUSY. Assim, em 1981108, os físicos norteamericanos Savas Dimoupolos (n.1952) (de origem grega) e Howard Mason Georgi (n.1947) apresentaram uma extensão da SUSY ao SM, o hoje conhecido Minimal Supersimetric Standard Model (MSSM), no qual se considera uma transformação com simetria global, isto é, que é aplicada uniformemente em todos os pontos do espaço, permitindo transformar bósons em férmions e vice-versa. Além do mais, ela prediz as partículas ino com massas entre $100 \mathrm{GeV}$ e $1 \mathrm{TeV}$. Contudo, as experiências realizadas (Bernardi, Carena and Junk, op. cit.), pelo Tevatron e pelo LEP e LHC do CERN, até 2013, envolvendo energias até a ordem de TeV e que indicaram a existência de $\mathrm{H}$ neutros, não encontraram nenhuma evidência das partículas SYSY/ino.

Para finalizar este artigo, é oportuno registrar que o LHC continuou a realizar experiências, por intermédio de duas colaborações:

1) LHCb, em um experimento envolvendo a oscilação entre o méson B_s [composto de um antiquark bonito e um quark estranho $(\bar{b} s)$ e cuja massa foi pela primeira vez medida, em $1993^{109}$, por D. Buskulic e Alexander Finch] e sua antipartícula $\left(\bar{B} \_\mathrm{s}\right)$;

\footnotetext{
106

Physics Letters B46, p. 109, 1973.

107

Physical Review Letters, v. 30, p. 716, 1973.

108

Nuclear Physics B193, p. 150, 1981.

109

Physics Letters B311, p. 425,1993.
} 
2) CMS, em busca das inos, sem obter sucesso, conforme artigo publicado, em setembro de $2013^{110}$, pelos físicos Jonathan L. Feng, Philipp Kant, Stefano Profume e David Sanford. Neste artigo, esses autores afirmam que, embora a violação da simetria CP (carga/paridade) observada no decaimento (B_s $\left.\rightarrow \mu^{+}+\mu^{-}\right)$ confirme o SM (com precisão $>4 \sigma$ ), porém não foi encontrado nenhum SUSY/ino como se esperava. Desse modo, alguns físicos afirmaram que essa experiência deixou ainda uma porta muita aberta para uma Nova Física ${ }^{111}$. Apesar dessa descrença, a esperança de confirmar o MSSM foi proposta, em outubro de $2013^{112}$, no artigo escrito por Carena, S. Heinemeyer, Oscar Stal, C. E. M. Wagner e G. Weiglein e intitulado: Benchmark Scenarios after the Discovery of a Higgs-like Particle.

\section{Agradecimentos}

Agradeço a leitura crítica deste artigo aos físicos brasileiros: Denis Oliveira Damazio, Gilvan Augusto Alves, José Guilherme Rocha de Lima (meu exaluno na Universidade Federal do Pará), Luciano Manhães de Andrade Filho, Sérgio Ferraz Novaes e Vitor Oguri.

${ }^{110}$ Physical Review Letters, v. 111, a.n. 131802, 2013.

111

Wikipedia/Strange B meson.

112

European Journal Physics C73, p. 2552, 2013. 